\title{
EARLY PREDICTION OF INTRACTABLE CHILDHOOD EPILEPSY
}

The early identification of children at risk for intractable epilepsy (IE) would facilitate a choice of more aggressive and effective medical or surgical methods of management. Children with newly diagnosed epilepsy were prospectively identified by participating pediatric neurologists in the State of Connecticut, Jan 1993 through Dec 1997. Parental interviews and follow-up medical record reviews and analyses were conducted by researchers at Montefiore Medical Center, Bronx, NY; and Yale and Northern Illinois Universities. Median follow up was 4.8 years, and $98 \%$ were followed for more than 18 months. Intractability was defined as failure of response to two or more drugs and at least one seizure per month over 18 months. Drugs were given in maximal tolerated doses and noncompliance was ruled out. Of a total of 613 newly diagnosed cases, $60(10 \%)$ met criteria for intractability. Of these, $35 \%$ were classified (according to International League Against Epilepsy guidelines) as cryptogenic/symptomatic generalized epilepsies, $2.7 \%$ as idiopathic, $11 \%$ as other localization-related, and $8 \%$ were unclassified. Risk of intractability was highly correlated with etiology and epilepsy syndrome $(p<0.001)$. The highest risk of IE was in the symptomatic group and the lowest in idiopathic cases. IE was also correlated with a high initial seizure frequency (an interval of $<6$ months vs. 4,7 months between 2 seizures) ( $p<0.0001)$; focal EEG slowing $(p=0.02)$; and acute symptomatic or neonatal status epilepticus $(\mathrm{p}=0.001)$. Age at onset between 5 and 9 years was associated with a lowered risk of IE $(p=0.03)$. Factors not significantly correlated with early intractability included age of onset less than 1 year, absolute number of seizures and duration of epilepsy, and unprovoked or febrile status epilepticus. (Berg AT, Shinnar S, Levy SR et al. Early development of intractable epilepsy in children. A prospective study. Neurology June (1 of 2) 2001;56:1445-1452). (Reprints: Anne T Berg PhD, Department of Biological Sciences, Northern Illinois University, DeKalb, IL 60115).

COMMENT. The authors have provided some valuable statistics on the risk factors involved in children with intractable epilepsy (IE) and a means of early predictability. The prognosis of IE may be improved by a more timely selection of cases for trials of newer antiepileptic drugs, and surgical or dietary methods of management. According to the International Classification of Epilepsy, epilepsies are grouped as localization-related (focal, local, partial), or generalized. Localization-related epilepsies are idiopathic/genetic (eg, benign childhood epilepsy with centrotemporal spikes (BECTS)) or symptomatic/cryptogenic (of known or unknown etiology). Generalized epilepsies are grouped as idiopathic, with age-related onset (eg. benign neonatal, benign myoclonic in infancy, childhood and juvenile absence), symptomatic, and idiopathic and/or symptomatic (eg. West's and Lennox-Gastaut syndromes). Idiopathic or primary forms of epilepsy usually carry a better prognosis for response to treatment than symptomatic/secondary epilepsies. As may be predicted, symptomatic generalized epilepsies, a high initial seizure frequency, and focal EEG findings correlate with a likely intractable course.

The certainty of predicting intractability based on these criteria is questioned by some authorities (Holmes GL, Engel J Jr. Neurology 2001;56:14301431). Is failure to respond after only 2 drugs sufficient for inclusion as IE, when $14 \%$ eventually were controlled within 1.5 and 3 years? A rush to surgical intervention based on these criteria may be inappropriate. May some patients initially responsive later develop intractability? Children with mesial temporal lobe epilepsy, most commonly treated surgically, are particularly susceptible to 
late onset intractability (Engel J Jr et al, 1997), a condition not addressed in this study. Despite these criticisms and cautionary comments, the criteria for IE identified by Berg and associates will be valuable in counseling parents on the increased necessity for adequate treatment and careful and frequent monitoring of seizures.

\section{DEMYELINATING DISORDERS}

\section{ACUTE DISSEMINATED ENCEPHALOMYELITIS}

The clinical and neuroradiological features of acute disseminated encephalomyelitis (ADEM) were determined by a retrospective review of medical records and MRI findings in 31 children (mean age 5.9 yrs; range 2-16 yrs) examined at the Royal Children's Hospital, Melbourne, Australia, between 1993 and 1998. Prodromal illnesses, mainly upper respiratory or nonspecific infections, occurred in 22 (71\%). Two had received hepatitis B vaccine 3 to 6 weeks prior to symptom onset. Neurologic symptoms, developing over a mean of 4 days, included ataxia (65\%), cranial nerve pareses (45\%), headache $(45 \%)$, vomiting $(35 \%)$, neck stiffness $(26 \%)$, impaired consciousness $(68 \%)$, seizures $(13 \%)$, optic neuritis $(13 \%)$, hemiparesis $(23 \%)$, and language disturbance (29\%). CSF protein was elevated in $50 \%(0.4-0.6 \mathrm{~g} / \mathrm{L})$, and csf white cell counts were abnormal, mainly

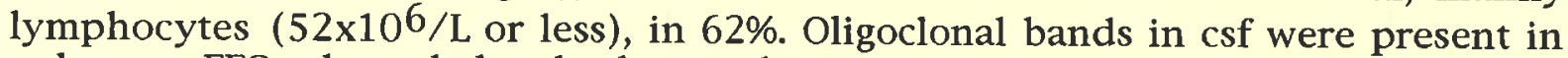
only one. EEGs showed slow background activity in 5 of 7 tested. Serologic testing showed elevated immunoglobulin $M$ titers to $M$. pneumoniae in 4, and to EpsteinBarr virus in 2. PCR was negative in csf of 23 tested. MRI showed bilateral, asymmetrical involvement of white matter of frontal and parietal lobes in all but 3 patients; the lesions resembled tumor masses in 2 with the diagnosis of demyelination confirmed by biopsy. Corpus callosum and periventricular lesions characteristic of MS were present in 29\%. Deep gray matter was involved in $61 \%$ and the spinal cord in 16\%. High-dose IV methylprednisolone was usually effective in treatment. Recovery was complete in $81 \%$, and sequelae were mild in the remainder. (Hynson JL, Kornberg AJ, Coleman LT et al. Clinical and neuroradiologic features of acute disseminated encephalomyelitis in children. Neurology May (2 of 2) 2001;56:1308-1312 ). (Respond: Dr Andrew J Kornberg, Department of Neurology, Royal Children's Hospital, Flemington Road, Parkville, Victoria, Australia 3052).

COMMENT. Serial MRI may be necessary to distinguish ADEM from MS in some cases. In the absence of a clinical relapse, new lesions should not appear in ADEM. The diagnosis of ADEM is usually apparent at presentation. Characteristic features are the prodromal viral illness, early onset of ataxia, large lesions on MRI with involvement of deep gray matter including thalamus, and absence of oligoclonal bands.

ADEM in a patient with autoimmune hemolytic anemia. A 16-year old girl with a 3 year history of hemolytic anemia had received immunosuppressive prednisolone treatment at Chang Gung Children's Hospital, Taoyuan, Taiwan. She developed a systemic infection with Cryptococcus neoformans which triggered the encephalomyelitis. Her symptoms included ataxia, drowsiness, and coma. MRI showed multiple bilateral, asymmetrical lesions involving subcortical white matter, basal ganglia, and frontoparietal lobes. She responded to IV immunoglobulin IVIG, the ataxia and weakness resolving after 2 weeks. Follow-up MRI in 1 month was almost normal, and neurologic symptoms had completely resolved. No relapse had occurred at 1 year follow-up. (Jaing T-H, Lin K-L, Chiu C- 